\title{
A quantum and semiclassical study of dynamical resonances in the $\mathrm{C}+\mathrm{NO} \rightarrow \mathrm{CN}+\mathrm{O}$ reaction
}

\author{
Ravinder Abrol \\ Arthur Amos Noyes Laboratory of Chemical Physics, California Institute of Technology, \\ Pasadena, California 91125 \\ Laurent Wiesenfeld ${ }^{\text {a) }}$ \\ Arthur Amos Noyes Laboratory of Chemical Physics and Control and Dynamical Systems, \\ California Institute of Technology, Pasadena, California 91125 \\ Bruce Lambert and Aron Kuppermann ${ }^{\text {b) }}$ \\ Arthur Amos Noyes Laboratory of Chemical Physics, California Institute of Technology, \\ Pasadena, California 91125
}

(Received 23 August 2000; accepted 19 December 2000)

\begin{abstract}
Accurate quantum mechanical reactive scattering calculations were performed for the collinear $\mathrm{C}+\mathrm{NO} \rightarrow \mathrm{CN}+\mathrm{O}$ reaction using a polynomial-modified London Eyring Polanyi Sato (PQLEPS) potential energy surface (PES), which has a $4.26 \mathrm{eV}$ deep well in the strong interaction region, and a reference LEPS PES, which has no well in that region. The reaction probabilities obtained for both PESs show signatures for resonances. These resonances were characterized by calculating the eigenvalues and eigenvectors of the collision lifetime matrix as a function of energy. Many resonances were found for scattering on both PESs, indicating that the potential well in the PQLEPS PES does not play the sole role in producing resonances in this relatively heavy atom system and that Feshbach processes occur for both PESs. However, the well in the PQLEPS PES is responsible for the differences in the energies, lifetimes, and compositions of the corresponding resonance states. These resonances are also interpreted in terms of simple periodic orbits supported by both PESs (using the WKB formalism), to further illustrate the role played by that potential well on the dynamics of this reaction. The existence of the resonances is associated with the dynamics of the long-lived CNO complex, which is much different than that of systems having an activation barrier. Although these results were obtained for a collinear model of the reaction, its collinearly-dominated nature suggests that related resonant behavior may occur in the real world. (C) 2001 American Institute of Physics. [DOI: 10.1063/1.1349083]
\end{abstract}

\section{INTRODUCTION}

The occurrence of dynamical resonances in quantum mechanical reactive scattering calculations of atom-diatomic molecule collisions has provided valuable insight into the understanding of chemical reactions. A common signature of these resonances is usually the rapidly changing state-to-state reaction probabilities as a function of energy. This was first observed in scattering calculations of the collinear $\mathrm{H}+\mathrm{H}_{2}$ reaction ${ }^{1,2}$ and later in the calculation of several other chemical reactions, ${ }^{3}$ including $\mathrm{F}+\mathrm{H}_{2}$ and its isotopic counterparts. These dynamical resonances are very sensitive to features of the potential energy surface (PES) that governs the nuclear dynamics, so their detailed analysis can provide a "spectroscopic tool" for the examination of transition states for chemical reactions. The experimental observation of resonances in the isotopic $\mathrm{F}+\mathrm{H}_{2}$ systems ${ }^{4,5}$ has increased the interest in these resonances.

Due to the effect of resonances on the dynamics of re-

\footnotetext{
a)Permanent address: Laboratoire de Spectrométrie Physique, Université Joseph-Fourier-Grenoble, France.

b) Author to whom correspondence should be addressed. Electronic mail: aron@caltech.edu
}

active collisions, numerous approximate techniques have been developed for understanding their origin, predicting the energies at which they occur, ${ }^{6,7}$ their widths, ${ }^{8}$ and their lifetimes. A method that has been shown ${ }^{9}$ to characterize the resonances unambiguously is based on the energy dependence of the eigenvalues of Smith's collision lifetime matrix. ${ }^{10}$ Its application to the $\mathrm{F}+\mathrm{H}_{2}$ reaction ${ }^{9}$ and its deuterium-substituted counterparts showed that only one eigenvalue changed rapidly with energy at the resonance position and it did so from a relatively flat background. This facilitated the calculation of resonance energies, their widths and their lifetimes. Similar conclusions were obtained for the $\mathrm{H}+\mathrm{H}_{2}$ system $^{11}$ on the scaled SSMK (Ref. 1) PES and for the $\mathrm{Cl}+\mathrm{HBr}$ system. ${ }^{12}$ These conclusions have also been shown to be valid for the two lowest energy resonances in the $\mathrm{H}+\mathrm{H}_{2}$ reaction $^{13}$ on the Porter-Karplus ${ }^{14}$ PES in three dimensions using only the $J=0$ partial wave as well as in the collinear case. ${ }^{15}$ They have been extended to the cross sections for $\mathrm{H}+\mathrm{D}_{2}$ accurately incorporating all partial waves. ${ }^{16}$ Abu-Salbi et al. ${ }^{17}$ have also considered the relationship between dynamical resonances, vibrationally adiabatic barriers, and quantal time delays in the collinear $\mathrm{H}+\mathrm{H}_{2}$ reaction. Many other systems exhibit a rich resonance structure in 
their reactive collision dynamics. Of particular interest here is the $\mathrm{He}+\mathrm{H}_{2}^{+}$system as well as its isotopic substitutions, studied by Sathyamurthy et al. ${ }^{18}$ In their series of papers, these authors proceeded into an extensive comparison between the quantum mechanical resonances and classical mechanics on the PES considered.

For many simple reactive collisions, an interpretation of the various resonances has been sought in a semiclassical framework. ${ }^{19-23}$ At various degrees of sophistication, one looks for a correlation between the resonances displayed by the quantum mechanical transition probability and the periodic orbits (p.o.s) of the classical system evolving on the same PES. Semiclassical quantization schemes of the classical dynamics have been used with success in several instances. ${ }^{24-28}$ They are all based on a careful study of the classical mechanics of the scattering or bound systems, as well as periodic orbit quantization. The scattering systems are subjected to a semiclassical quantization in a manner analogous - though not fully equivalent- to the semiclassical quantization of energy levels for bound systems. In general, it is hoped that at energies $E_{q}$ where a p.o. quantizes, a pole will appear in the S-matrix, leading to a resonance. For very simple systems, from a dynamical point of view (e.g., in scattering of disks), a very sophisticated version of this simple idea has been shown to be valid. ${ }^{29}$

In this study, we consider the reaction

$$
\mathrm{C}\left({ }^{3} P\right)+\mathrm{NO}\left(X^{2} \Pi\right) \rightarrow \mathrm{CN}\left(X^{2} \Sigma^{+}\right)+\mathrm{O}\left({ }^{3} P\right) .
$$

As it involves three relatively heavy atoms, it has been the focus of attention for some time now. An $a b$ initio calculation of its PES by Halvick et al. ${ }^{30}$ was followed by several analytical fits and classical dynamics studies. ${ }^{31-34}$ Subsequent investigations include a collinear quantum wave packet calculation $^{35}$ to analyze the influence of the topology of the potential well on the collinear dynamics, a statistical study ${ }^{36}$ to simulate the energy partitioning and rovibrational distribution of the $\mathrm{CN}$ molecule in crossed molecular beam experiments, and an estimation of the total rate coefficient ${ }^{37}$ using a capture approach based upon the long-range part of the potential and including spin-orbit coupling. A more accurate PES was calculated by Persson et al. ${ }^{38}$ which was followed by a rovibrational spectral analysis ${ }^{39}$ of the linear triatomic molecules $(\mathrm{CNO}, \mathrm{CON}$, and $\mathrm{NCO})$ and another $a b$ initio calculation by Simonson et al. ${ }^{40}$ Most recent studies include a two-degree-of-freedom geometrical investigation ${ }^{41}$ involving periodic orbits for the collinear $\mathrm{CNO}$ dynamics, a quasiclassical trajectory calculation ${ }^{42}$ over a wide temperature range, and a three-dimensional time-dependent wavepacket study ${ }^{43}$ for total angular momentum $J=0$.

In this paper, we present the results of accurate quantum mechanical reactive scattering calculations for the collinear $\mathrm{C}+\mathrm{NO} \rightarrow \mathrm{CN}+\mathrm{O}$ reaction, in which the state-to-state reaction probabilities as a function of energy and a detailed collision lifetime analysis of resonances, their energies and asymptotic compositions are presented for the first time for this system. Even though these calculations are for a collinear model, their results should be an indication of what happens in the 3D world, given that the PES for this system is collinearly dominated. In the $\mathrm{H}+\mathrm{H}_{2}$ and $\mathrm{F}+\mathrm{H}_{2}$ systems and (a) PQLEPS

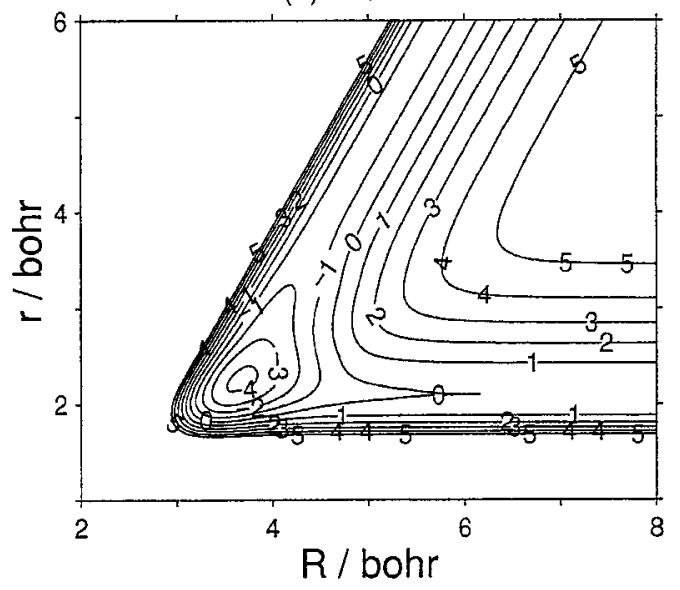

(b) LEPS

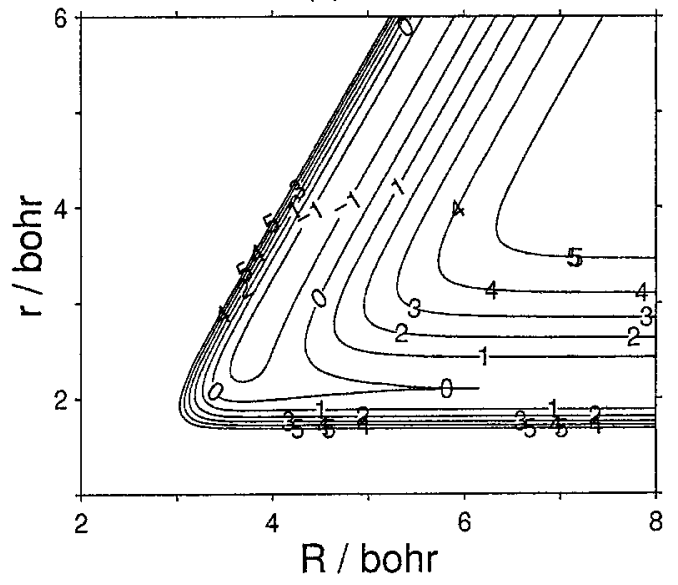

FIG. 1. Energy contours (in eV) for the two potential energy surfaces used, with $0 \mathrm{eV}$ being the bottom of the $\mathrm{C}+\mathrm{NO}$ channel. (a) PQLEPS PES. (b) LEPS PES.

their isotopic counterparts, the presence of resonances, first detected in collinear quantum calculations, also occurred in the individual partial waves of 3D calculations, as well as in the state-to-state differential cross sections calculated using enough partial waves for these cross sections to be converged. Furthermore, the mechanisms for resonant state formation inferred from the collinear calculations were also operative in the $3 \mathrm{D}$ reactions. An analogous situation may prevail for the $\mathrm{C}+\mathrm{NO}$ system. Because of the heavy nature of the atoms in this system compared to $\mathrm{H}, \mathrm{D}$, and $\mathrm{F}$, the number of channels needed for a converged $3 \mathrm{D}$ calculation is significantly higher. As a result, before embarking on such a calculation, it is not only desirable but also prudent to be guided by the collinear results presented here.

In order to determine the effect of the features of PESs on reactive collisions and resonances, a polynomial-modified London Eyring Polanyi Sato (PQLEPS) PES (Ref. 31) and a reference LEPS PES (Ref. 32) having the same asymptotic characteristics were used (see Fig. 1). Both PESs have an exothermicity of $1.35 \mathrm{eV}$. The main difference between them is that the PQLEPS PES [Fig. 1(a)] has a $4.26 \mathrm{eV}$ deep potential well in the strong interaction region [relative to the bottom of the reagent $(\mathrm{C}+\mathrm{NO})$ channel], whereas the LEPS 
(a) PQLEPS
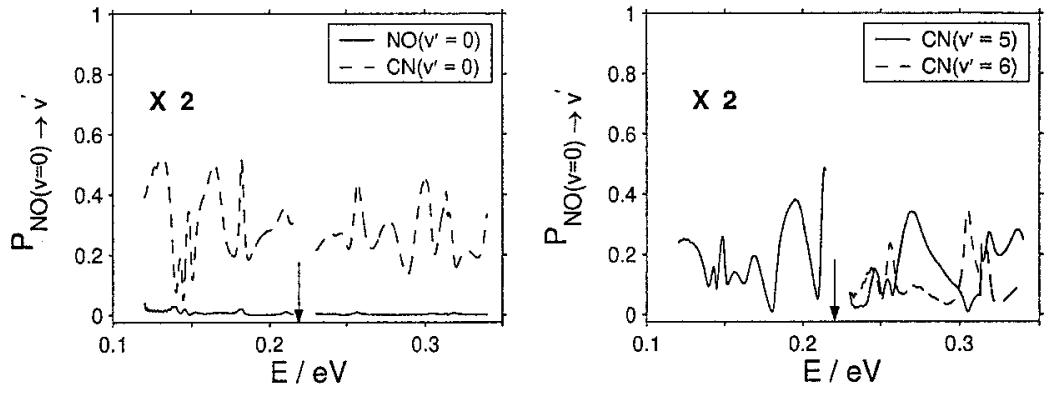

(b) LEPS
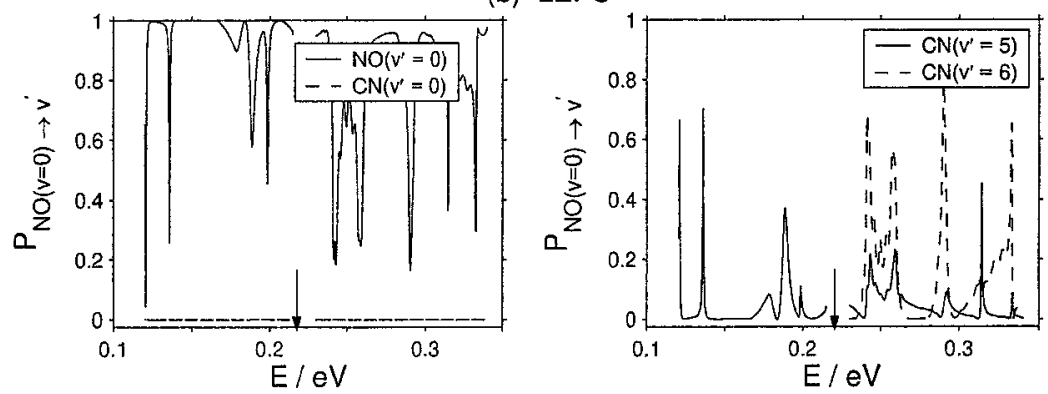

FIG. 2. State-to-state transition probabilities $P_{0 \rightarrow v^{\prime}}$ as a function of total energy $E(\mathrm{eV})$. The initial state for all these curves is $\mathrm{NO}(v=0)$. The final state is either NO or $\mathrm{CN}$, in state $v^{\prime}$, as indicated in the upper right-hand side rectangles. The arrow at $0.22 \mathrm{eV}$ locates the opening of the $\mathrm{CN}\left(v^{\prime}=6\right)$ channel. (a) PQLEPS PES. (b) LEPS PES. The $\times 2$ in the PQLEPS plots indicates the factor by which the corresponding probabilities were multiplied before plotting.
PES [Fig. 1(b)] has no potential well in that region. This PQLEPS PES well depth is close to those of the two more recent surfaces, $4.25 \mathrm{eV}$ (Ref. 40) and $4.21 \mathrm{eV},{ }^{42}$ respectively, and furnishes a satisfactory representation of the regions of congifuration space sampled in our studies.

In Sec. II, we present the quantum and semiclassical methodologies used and in Sec. III we describe and discuss the results obtained. In Sec. IV, a summary and conclusions are given.

\section{METHODOLOGY}

Both the quantum and semiclassical calculations presented here are based on two analytical PESs, the PQLEPS PES (Ref. 31) and a simpler LEPS PES. ${ }^{32}$ As is well known, ${ }^{3}$ the Hamiltonian of the collinear triatomic system is given by

$$
H=\frac{1}{2 \mu}\left(p_{r}^{2}+p_{R}^{2}\right)+V(r, R),
$$

where $\mu=\left(m_{\mathrm{C}} m_{\mathrm{N}} m_{\mathrm{O}} / M\right)^{1 / 2}$ is the reduced mass of the system, $M$ is its total mass, and $m_{X}(X=\mathrm{C}, \mathrm{N}, \mathrm{O})$ are the masses of the three atoms. The Jacobi coordinates $r$ and $R$ are massscaled with respect to the physical distances $R^{\prime}$ (of $\mathrm{C}$ to the center-of-mass of NO) and $r^{\prime}$ (between $\mathrm{N}$ and $\mathrm{O}$ ) according to

$$
R=a R^{\prime}, r=a^{-1} r^{\prime}, a=\left(\frac{\mu_{\mathrm{C}, \mathrm{NO}}}{\mu_{\mathrm{NO}}}\right)^{1 / 4},
$$

where $p_{r}$ and $p_{R}$ are the associated momenta and $V(r, R)$ the system's potential energy. The Schrödinger or Hamilton equations are derived from that Hamiltonian.

\section{A. Quantum mechanical formalism and numerical parameters}

The quantum calculations were done using hyperspherical coordinates, ${ }^{44}$ expanding the time-independent scattering wave function in local hyperspherical surface functions, and solving the resulting coupled channel equations by a logarithmic derivative method from small to large values of the hyperradius. From these solutions the scattering matrix $S$ was obtained.

The collision lifetime matrix $Q$ has been defined by Smith $^{10}$ and is related to the open part $S_{0}$ of $S$ by the expression

$$
\mathrm{Q}=-i \hbar \mathrm{S}_{0} \frac{d \mathrm{~S}_{0}^{\dagger}}{d E} .
$$

Since $S_{0}$ is unitary and symmetric, $Q$ is Hermitian, its eigenvalues are real and its eigenvector matrix is unitary. All these quantities depend on the system's total energy $E$. These eigenvalues are interpreted as the lifetimes of the long-lived metastable states associated with the resonances. The wave functions that describe these states are obtained from the corresponding eigenvectors of $Q$.

Two sets of calculations were performed, one for the PQLEPS PES and another for the LEPS PES. The corresponding number of basis functions were 26 and 14, respectively. The unitarity of the $S_{\circ}$ matrices was $1 \pm 0.01$ or better. The two sets of scattering matrices were obtained on a dense energy grid $(0.1 \mathrm{meV})$ of 2201 energies in the total energy range of $0.12 \mathrm{eV}$ to $0.34 \mathrm{eV}$ (measured with respect to the bottom of the isolated NO diatom potential energy curve, corresponding to translation energies of $\mathrm{C}$ with respect to ground state $\mathrm{NO}$ of $0.003 \mathrm{eV}$ to $0.223 \mathrm{eV}$ ). This high grid density permitted the use of a three-point finite difference method for the calculation of the energy derivatives. The resulting $Q$ was approximately Hermitian and was fully hermitianized by replacing it by an average of itself and its Hermitian adjoint.

\section{B. Semiclassical description}

It has been often argued that interesting insights of some quantum dynamical features such as resonances, may be ob- 
tained from classical dynamics. The Hamiltonian is understood as the generator of either a quantum mechanical flow or else a classical Hamiltonian flow, whose characteristics (trajectories, periodic orbits) are examined in order to perform a semiclassical quantization. ${ }^{45}$ The main tool that allows us to find semiclassical approximations of energy levels or resonances are the periodic orbits (p.o.) of the classical dynamics.

If the system were bound with one degree of freedom, a Wentzel-Kramers-Brillouin (WKB) semiclassical quantization of the spectrum would be a natural first approximation,

$$
I_{\gamma}=\oint_{\gamma} p d q=h(n+1 / 2),
$$

where $I_{\gamma}$ is the action of the p.o. $\gamma$, described by coordinate $q$ and momentum $p$. The integer $n$ characterizes the level number, and hence the number of nodes of the onedimensional wave function $\psi(q)$, in a coordinate representation. If there is more than one degree of freedom-there are two for the present case-the WKB formalism is not applicable. If the system is bound and fully integrable, the Einstein-Brillouin-Keller (EBK) quantization scheme ${ }^{45}$ (or some variation of it, such as the Berry-Tabor formula ${ }^{46}$ ) provides a very good approximation of the bound spectrum (see Ref. 28 for a recent example). Spectra of fully chaotic systems may also be derived from semiclassical quantization, with the help of Gutzwiller or Gutzwiller-Voros formulas; ${ }^{45,47,48}$ atomic hydrogen in a magnetic field was successfully quantized this way. ${ }^{49}$

Resonances of scattering systems, seen as poles of the $\mathrm{S}(E)$ matrix, can also be obtained from the p.o.s of the unbound systems. These p.o.s are of finite extension, that is, they represent the vibrational motion of the possibly short- or long-lived intermediate complex. Some simple models of scattering systems also allow for a full semiclassical interpretation of their resonances, like the 3-disk model ${ }^{29}$ or a more general model of a potential consisting of a tube and an interaction region. ${ }^{50}$ However, the situation in the present $\mathrm{C}+\mathrm{NO}$ scattering system is somewhat more involved, because of the simultaneous presence of chaotic regions and regular regions in phase space. Because of the heavy masses ( $\mu=8 \mathrm{amu}$ ), neither the chaotic regions nor the regular regions of phase space may be neglected. Our objectives will consequently be more limited and only main resonances and main p.o.s will be considered.

We resort thus to a simpler, naive semiclassical scheme, allowing for the various p.o. to be quantized in a WKB fashion, but not allowing for any interference between those orbits. It has been shown that it is enough, for our very simple purposes, ${ }^{25,26}$ to simply use a WKB formula, with a correct zero-point energy (or Maslov angle ${ }^{45,51}$ ), to find the approximate energies of the main resonances. Since we are dealing with an open system, the dynamics explores the phase space structure in the strong interaction region only for a short amount of time (of the order of $100 \mathrm{fs}$, that is, a few vibrational periods). In this fashion, we expect that complicated interference effects between various periodic orbits have no time to develop. Hence, the simple-minded approach is equivalent to taking only the first few terms of the Gutzwiller
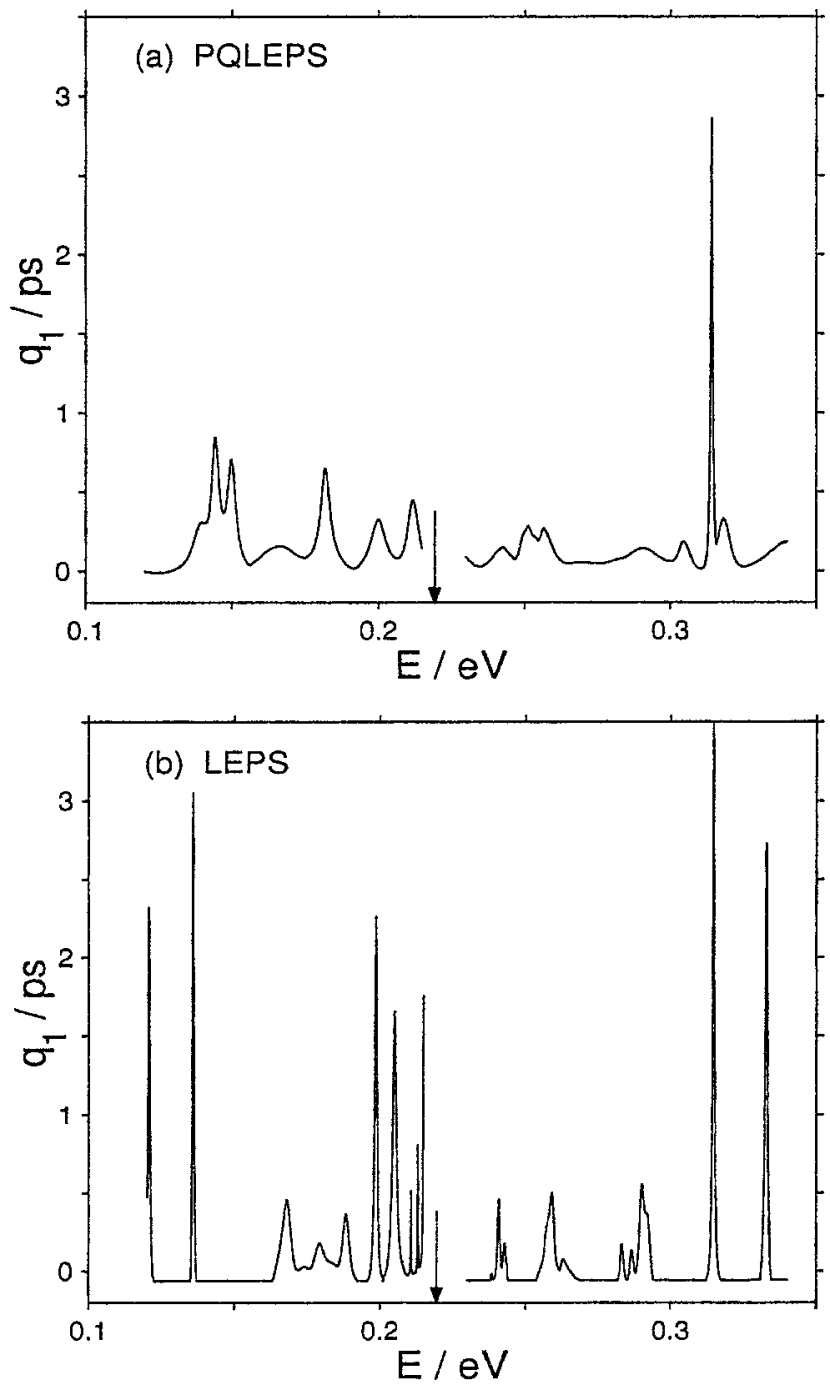

FIG. 3. Largest eigenvalue $q_{1}$ (in ps) of Smith's collision lifetime matrix $Q$ as a function of $E$ (in eV). (a) PQLEPS PES. (b) LEPS PES.

or Berry-Tabor formulas, excluding all cross-terms. It is expected that better precision should be obtained for shortlived resonances than for long-lived ones.

We thus follow the main periodic orbits $\gamma_{i}$ of the PQLEPS and LEPS PESs as they change with energy, and calculate the integrals,

$$
I_{\gamma_{i}}(E)=\int_{\gamma_{i}} p_{\gamma_{i}} d q_{\gamma_{i}}
$$

where coordinates and momenta are taken along the p.o. Since all the p.o.s found are of the vibration type (as we are considering a collinear system), the quantization condition is given by Eq. (4).

\section{RESULTS AND DISCUSSION}

\section{A. Quantum mechanical results}

Potential energy contour diagrams for the PQLEPS and LEPS PESs of the $\mathrm{C}+\mathrm{NO}$ system, are depicted in Fig. 1. The state-to-state reaction probabilities are obtained by taking the absolute-value-squared of the corresponding elements of the scattering matrices. In what follows, $P_{0 v^{\prime}}^{\mathrm{R}}$ and $P_{0 v^{\prime}}^{\mathrm{NR}}$ repre- 
TABLE I. Resonance energies, collision lifetimes, and compositions for the PQLEPS PES.

\begin{tabular}{|c|c|c|c|c|c|c|c|c|}
\hline \multicolumn{9}{|c|}{ Resonance energy / $\mathrm{eV}^{\mathrm{a}}$} \\
\hline & 0.1443 & 0.1498 & 0.1819 & 0.1999 & 0.2119 & 0.2512 & 0.2567 & 0.3142 \\
\hline \multicolumn{9}{|c|}{ Collision lifetime / $\mathbf{p s}^{\mathrm{b}}$} \\
\hline & 0.846 & 0.703 & 0.648 & 0.327 & 0.449 & 0.283 & 0.267 & 2.857 \\
\hline \multicolumn{9}{|c|}{ Composition $^{c}$} \\
\hline $\mathrm{NO}(v=0)$ & 0.02 & 0.02 & 0.05 & 0.00 & 0.03 & 0.03 & 0.02 & 0.03 \\
\hline $\mathrm{CN}\left(v^{\prime}=0\right)$ & 0.40 & 0.35 & 0.14 & 0.09 & 0.01 & 0.09 & 0.10 & 0.02 \\
\hline $\mathrm{CN}\left(v^{\prime}=1\right)$ & 0.04 & 0.04 & 0.02 & 0.04 & 0.12 & 0.25 & 0.24 & 0.11 \\
\hline $\mathrm{CN}\left(v^{\prime}=2\right)$ & 0.31 & 0.29 & 0.24 & 0.38 & 0.09 & 0.17 & 0.18 & 0.15 \\
\hline $\mathrm{CN}\left(v^{\prime}=3\right)$ & 0.12 & 0.15 & 0.17 & 0.19 & 0.11 & 0.15 & 0.07 & 0.17 \\
\hline $\mathrm{CN}\left(v^{\prime}=4\right)$ & 0.03 & 0.00 & 0.26 & 0.17 & 0.15 & 0.03 & 0.13 & 0.14 \\
\hline $\mathrm{CN}\left(v^{\prime}=5\right)$ & 0.08 & 0.15 & 0.11 & 0.14 & 0.49 & 0.21 & 0.11 & 0.15 \\
\hline $\mathrm{CN}\left(v^{\prime}=6\right)$ & 0.00 & 0.00 & 0.00 & 0.00 & 0.00 & 0.06 & 0.16 & 0.21 \\
\hline Total & 1.00 & 1.00 & 0.99 & 1.01 & 1.00 & 0.99 & 1.01 & 0.98 \\
\hline
\end{tabular}

${ }^{a}$ Energies at which the collision lifetimes in Fig. 3(a) show a maximum.

${ }^{b}$ Largest eigenvalue of the collision lifetime matrix $Q$ at the peaks of Fig. 3(a).

${ }^{\mathrm{c}}$ Square of the absolute value of the the eigenvector elements corresponding to the largest eigenvalue of $\mathrm{Q}$.

sent, respectively, the probabilities of the $\mathrm{C}+\mathrm{NO}(v=0)$ $\rightarrow \mathrm{CN}\left(v^{\prime}\right)+\mathrm{O}$ and $\mathrm{C}+\mathrm{NO}(v=0) \rightarrow \mathrm{C}+\mathrm{NO}\left(v^{\prime}\right)$ transitions. Several such probabilities are shown in Figs. 2(a) (PQLEPS, with a scaling factor of 2) and 2(b) (LEPS). The four transitions shown are typical and highlight the differences due to the different potential surfaces used to obtain them. All transitions for both PESs show rapid variations with energy, providing the first signature of the presence of many resonances in this system. The arrow at about $0.22 \mathrm{eV}$ indicates the threshold energy of the $\mathrm{C}+\mathrm{NO}(0) \rightarrow \mathrm{CN}(6)+\mathrm{O}$ process.

For the PQLEPS PES (having a $4.26 \mathrm{eV}$ deep well in the strong interaction region), the nonreactive reaction probability $P_{00}^{\mathrm{NR}}$ remains close to zero over the full energy range displayed in Fig. 2(a). Over that same range, the reactive transition probability $\left(P_{00}^{\mathrm{R}}\right)$ has an average of about 0.15 and changes rapidly between about 0.05 and about 0.25 . The $P_{05}^{\mathrm{R}}$ and $P_{06}^{\mathrm{R}}$ transitions also vary rapidly over this energy range staying under 0.25 . For the LEPS PES (with no potential well in the strong interaction region), the nonreactive transition probability, stays above 0.90 over most of the energy range except for a few narrow ranges, in which it sharply drops below 0.90 and sometimes is as low as 0.10 [see Fig. 2(b)]. These sharp drops in $P_{00}^{\mathrm{NR}}$ are complemented by sharp increases in $P_{05}^{\mathrm{R}}$ below $0.22 \mathrm{eV}$ and in both $P_{05}^{\mathrm{R}}$ and $P_{06}^{\mathrm{R}}$ beyond $0.22 \mathrm{eV}$, since the $\mathrm{CN}\left(v^{\prime}=6\right)$ channel opens at this energy. Over the full energy range $(0.12 \mathrm{eV}-0.34 \mathrm{eV}), P_{00}^{\mathrm{R}}$ is close to zero, indicating that the $\mathrm{CN}\left(v^{\prime}=0\right)$ reaction product is not produced for the LEPS PES in this energy range because of the early energy release characteristic of this PES.

Although the transition probabilities on both PESs vary rapidly with energy, those for the LEPS PES vary much more sharply than those for the PQLEPS PES. Both show a signature for the presence of resonances and the differences in the behavior of their transition probabilities may be assigned to the deep well in the interaction region for the PQLEPS PES.

Smith's collision lifetime matrices and their eigenvalues and eigenvectors over the energy range $0.12 \mathrm{eV}-0.34 \mathrm{eV}$ were obtained for the two PESs. The largest eigenvalue $q_{1}$ is shown respectively for the PQLEPS and LEPS PESs in Figs. 3(a) and 3(b). Both PESs seem to produce similar numbers of resonances over the total energy range considered, although they have very different collision lifetimes. For the PQLEPS PES, only one resonance has a lifetime of more than 1 ps (at $2.857 \mathrm{ps),} \mathrm{whereas} \mathrm{for} \mathrm{the} \mathrm{LEPS} \mathrm{PES,} \mathrm{seven}$ resonances have a lifetime of more than $1 \mathrm{ps}$ (ranging from $1.5 \mathrm{ps}$ to $3.5 \mathrm{ps}$ ). The presence of a large number of longlived resonances for the LEPS PES, which has no potential well in the strong interaction region, indicates that a deep well is not essential for the occurrence of resonances in this heavy atom system. The fact that the LEPS resonances are in average longer-lived than the PQLEPS ones indicates that the deep well in the latter shortens the lifetime of the resonances, a rather surprising result. This is attributed at the end of Sec. IIIC to possible interferences between shape and internal excitation mechanisms for the PQLEPS PES. For the LEPS PES, the absence of a deep well precludes the occurrence of strong shape processes and therefore excludes such possible interferences.

From Figs. 3(a) and 3(b), we picked eight and eleven most long-lived resonances for the PQLEPS and LEPS PESs, respectively. We list the corresponding eigenvector compositions associated with $q_{1}$ in Tables I and II (for PQLEPS and LEPS PESs, respectively). In both Tables I and II, the contributions for channels that contribute $5 \%$ or more have been highlighted in bold to indicate major contributions. For example, in Table II, for the resonance at $0.1359 \mathrm{eV}$, which has a lifetime of $3.046 \mathrm{ps}$, the NO $(v=0)$ channel contributes $75 \%$ and the $\mathrm{CN}\left(v^{\prime}=5\right)$ channel $23 \%$ to the eigenvector corresponding to this resonance state. All the rest of the channels contribute a total of $2 \%$. For the PQLEPS resonances (see Table I) four to seven channels are major contributors to the eigenvectors and no one channel contributes more than $50 \%$. This is in marked contrast with the LEPS resonances, where only two or three channels were major contributors and for all resonances except two (one at 0.1206 
TABLE II. Resonance energies, collision lifetimes, and compositions for the LEPS PES.

\begin{tabular}{|c|c|c|c|c|c|c|c|c|c|c|c|}
\hline \multicolumn{12}{|c|}{ Resonance energy / eV } \\
\hline & 0.1206 & 0.1359 & 0.1680 & 0.1883 & 0.1985 & 0.2051 & 0.2150 & 0.2592 & 0.2901 & 0.3147 & 0.3329 \\
\hline \multicolumn{12}{|c|}{ Collision lifetime / $\mathrm{ps}^{\mathrm{b}}$} \\
\hline & 2.320 & 3.046 & 0.455 & 0.361 & 2.263 & 1.659 & 1.757 & 0.499 & 0.551 & 3.487 & 2.728 \\
\hline \multicolumn{12}{|c|}{ Composition $^{c}$} \\
\hline $\mathrm{NO}(v=0)$ & 0.39 & 0.75 & 0.00 & 0.87 & 0.83 & 0.00 & 0.01 & 0.25 & 0.28 & 0.17 & 0.22 \\
\hline $\mathrm{CN}\left(v^{\prime}=0\right)$ & 0.00 & 0.00 & 0.00 & 0.00 & 0.00 & 0.00 & 0.00 & 0.00 & 0.00 & 0.00 & 0.00 \\
\hline $\mathrm{CN}\left(v^{\prime}=1\right)$ & 0.00 & 0.00 & 0.00 & 0.00 & 0.00 & 0.00 & 0.00 & 0.00 & 0.00 & 0.00 & 0.00 \\
\hline $\mathrm{CN}\left(v^{\prime}=2\right)$ & 0.03 & 0.00 & 0.01 & 0.00 & 0.02 & 0.01 & 0.01 & 0.00 & 0.01 & 0.01 & 0.00 \\
\hline $\mathrm{CN}\left(v^{\prime}=3\right)$ & 0.15 & 0.01 & 0.03 & 0.00 & 0.07 & 0.03 & 0.05 & 0.00 & 0.02 & 0.04 & 0.01 \\
\hline $\mathrm{CN}\left(v^{\prime}=4\right)$ & 0.02 & 0.01 & 0.12 & 0.01 & 0.06 & 0.20 & 0.25 & 0.01 & 0.01 & 0.02 & 0.01 \\
\hline $\mathrm{CN}\left(v^{\prime}=5\right)$ & 0.40 & 0.23 & 0.83 & 0.11 & 0.02 & 0.75 & 0.68 & 0.36 & 0.12 & 0.70 & 0.06 \\
\hline $\mathrm{CN}\left(v^{\prime}=6\right)$ & 0.00 & 0.00 & 0.00 & 0.00 & 0.00 & 0.00 & 0.00 & 0.38 & 0.55 & 0.05 & 0.70 \\
\hline Total & 0.99 & 1.00 & 0.99 & 0.99 & 1.00 & 0.99 & 1.00 & 1.00 & 0.99 & 0.99 & 1.00 \\
\hline
\end{tabular}

${ }^{a}$ Energies at which the collision lifetimes in Fig. 3(b) show a maximum

${ }^{b}$ Largest eigenvalue of the collision lifetime matrix $Q$ at the peaks of Fig. 3(b).

${ }^{\mathrm{c}}$ Square of the absolute value of the the eigenvector elements corresponding to the largest eigenvalue of $\mathrm{Q}$.

$\mathrm{eV}$ and the other at $0.2592 \mathrm{eV}$ ) a single channel contributed more than $50 \%$ to the resonance. This indicates that the potential well in the PQLEPS PES has an effect on the resonance states of mixing more channels as compared to the well-devoid LEPS PES. The signature for the LEPS resonance at $0.1359 \mathrm{eV}$ (see Table II), for which the only contributions came from the $\mathrm{NO}(v=0)$ and $\mathrm{CN}\left(v^{\prime}=5\right)$ channels, is evident in the state-to-state reaction probability plot [see Fig. 2(b)], where the $P_{00}^{\mathrm{NR}}$ curve $\left[\mathrm{NO}\left(v^{\prime}=0\right)\right]$ drops sharply and the $P_{05}^{\mathrm{R}}$ curve $\left[\mathrm{CN}\left(v^{\prime}=5\right)\right]$ rises sharply at this energy $(0.1359 \mathrm{eV})$ confirming the contributions of these channels to the resonance state. A similar analysis can be made for the resonance at $0.2901 \mathrm{eV}$. It has a lifetime of $0.551 \mathrm{ps}$ and the composition of its eigenvector is $28 \%$ from the NO (0) channel, $12 \%$ from the $\mathrm{CN}$ (5) channel and 55\% from the CN (6) channel. The transition probability plot [Fig. 2(b)] shows that the reaction probabilities corresponding to these three channels drop and/or rise sharply at this energy confirming their contributions to its resonance state. The two PESs have a number of resonances and it is hard to correlate resonances from one PES with resonances from another because the resonance energies are different. But one resonance, with the largest collision lifetimes for both PESs, seems to be correlated. For the PQLEPS PES, it appears at $0.3142 \mathrm{eV}$ (collision lifetime of $2.857 \mathrm{ps}$ ) and for the LEPS PES at $0.3147 \mathrm{eV}$ (collision lifetime of $3.487 \mathrm{ps}$ ). Their positions differ by only $0.5 \mathrm{meV}$. The major difference between these two resonances is the composition of their eigenvectors. The PQLEPS resonance eigenvector has the composition: $11 \% \mathrm{CN}(1), 15 \% \mathrm{CN}(2), 17 \% \mathrm{CN}(3), 14 \% \mathrm{CN}(4)$, $15 \% \mathrm{CN}(5)$, and $21 \% \mathrm{CN}(6)$ and the LEPS resonance eigenvector has the composition: $17 \% \mathrm{NO}(0), 70 \% \mathrm{CN}(5)$, and 5\% $\mathrm{CN}$ (5). This observation again highlights the effect of the potential well in the PQLEPS PES that tends to mix the channels in the resonance states.

\section{B. Semiclassical results}

A study of the classical dynamics of the PQLEPS and LEPS PESs was undertaken, at total energies ranging from
$0.12 \mathrm{eV}$ to $0.34 \mathrm{eV}$, over an energy grid of $0.02 \mathrm{eV}$. The aim of this study was to follow the main p.o.s as they change with energy, undergoing possible bifurcations. The p.o.s were looked for firstly by a two-dimensional search on a Poincaré section (the $r=R$ line), then on two onedimensional searches, along the two equipotential lines $V$ $=E$. It must be noted that no p.o. of sufficiently low Lyapunov exponent ${ }^{19}$ (small instability) was found, that does not touch the $V=E$ line(s). Otherwise stated, all p.o.s found are self-retracing.

Among the p.o.s, one is similar in both the LEPS and the PQLEPS PES, namely, the antisymmetric stretch. Like in other cases studied, ${ }^{52}$ the antisymmetric stretch is a stable periodic orbit. It undergoes no bifurcation in the limited energy range studied and remains stable. On the other hand, all of the other simple p.o.s found were unstable. Some underwent bifurcations even in this very limited energy range. Figure 4 shows the variation of the action of each of the main p.o.s with energy, Eq. (5). They are all depicted in their respective domains of existence. Figure 4 shows the variation of the action $I(E)-I_{0}$, with different origins of action $I_{0}$ for all of them, as a matter of convenience. All the p.o.s found satisfy several times the quantization condition, $I(E)$ $=h(n+1 / 2)$, once for each value of $n$ as depicted in Fig. 4 . It must be stressed that because of the deep well, the absolute value of the actions are high; for the periodic orbit dividing surface (pods) (p.o. 0), $I_{0}=19$; the corresponding wave function has thus 18 nodes at the semiclassical quantization energy. This is compatible with the number of bound states supported by the system's well having a depth of about 2.9 $\mathrm{eV}$ (relative to the product $\mathrm{CN}+\mathrm{O}$ ) and a moderate anharmonicity. In Fig. 5, the corresponding periodic orbits are depicted, for an energy of $0.16 \mathrm{eV}$. Also, as has been already noted in Ref. 41, no p.o. corresponds to the symmetric stretch, because of the conjugate effect of the well and the exoergicity.

A similar study was undertaken for the LEPS PES. Results are roughly comparable, but at least one other stable p.o. was found, namely the symmetric stretch mode. This 


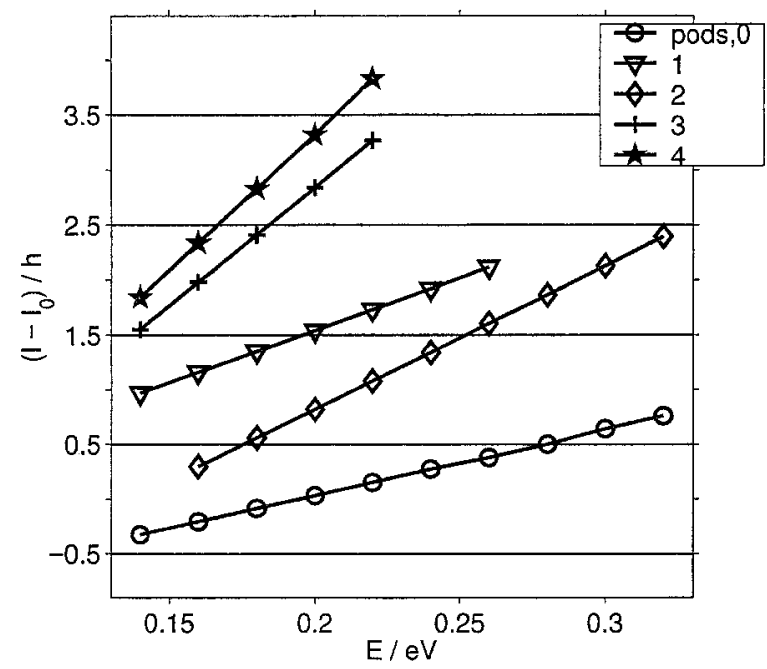

FIG. 4. Evolution of the actions $I(E)-I_{0}$ (in units of $h$ ), for the different periodic orbits of the PQLEPS PES, labeled by their number 0-4 (see Fig. 5). For sake of clarity, the origins have been displaced: $I_{0}(\mathbf{0})=19, I_{0}(\mathbf{1})$ $=30, I_{0}(2)=40, I_{0}(3)=66$, and $I_{0}(4)=68$.

symmetric mode is stable (and has no bifurcation) in the energy range under consideration. The two stable p.o.s are shown in Fig. 6. The semiclassical quantization was carried out in a manner similar to that for the PQLEPS PES. Because of the shape of the PES, many p.o.s are candidates for the various resonances depicted in Fig. 3(a). However, a group of two resonances stand out, with a nearly constant spacing of $0.0165 \mathrm{eV}$; they are discussed in the next section.

\section{Discussion of quantum and semiclassical results}

As was stressed earlier (Sec. II B), the semiclassical method employed here is the simplest possible one. In Figs. 7 and 8 we display the semiclassical resonance energies as a function of the quantum ones, for the PQLEPS and LEPS PES, respectively. As Fig. 7 shows, the agreement between these energies for the former is quite reasonable in spite of this method's simplicity. It must be emphasized that in this PQLEPS case, no attempt was made to choose among the various resonances in the quantum regime those that were to be reproduced semiclassically. The semiclassical quantization followed from an attempt of finding all p.o.s that were stable or mildly unstable (that is, whose instability is such that a precision of $10^{-4}$ bohr in the initial conditions is enough to define it). This is justified by recalling that the system under study is open, so that it has only a finite time to explore the classical dynamics. This leads us also to justify the better agreement with the quantum resonance energies (to within about $0.002 \mathrm{eV}$ ) for the short-lived $0.267 \mathrm{ps}$ one at $0.2567 \mathrm{eV}$, than for the long-lived ones (to within about $0.035 \mathrm{eV}),{ }^{25,46}$ such as the $2.857 \mathrm{ps}$ one at $0.3142 \mathrm{eV}$. While the latter's attribution to the antisymmetric stretch is clear cut, the pure first order WKB quantization is of course too rudimentary.

The LEPS PES (Fig. 8) offers a more difficult challenge, because of the larger number of p.o.s and of long-lived resonances. We concentrated thus only on one unambiguous series of resonances, separated by about $0.0165 \mathrm{eV}$, and all
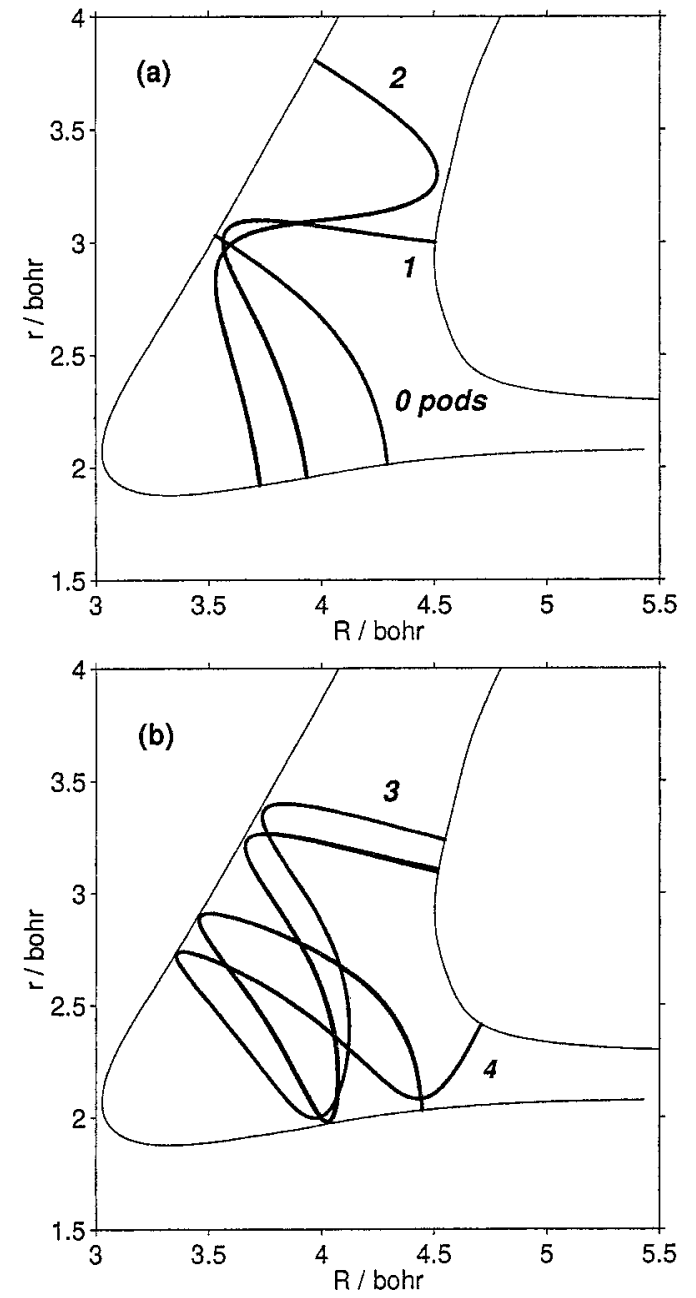

FIG. 5. The main periodic orbits of the PQLEPS PES, at an energy of 0.16 $\mathrm{eV}$, along with the relevant equipotentials $V=0.16 \mathrm{eV} . r$ and $R$ are the mass-scaled Jacobi coordinates. The $\mathbf{0}-\mathbf{4}$ numbers are the same as those in Fig. 4. The stable periodic orbit is labeled $\mathbf{0}$; it is a simple pods (periodic orbit dividing surface) in the sense of Ref. 41. It behaves like an antisymmetric stretch.

with a lifetime above $1.5 \mathrm{ps}$. This series may be easily attributed to the two primary stable periodic orbits, the symmetric and antisymmetric stretches. These two periodic orbits are shown in Fig. 6 and the agreement with the quantum resonances is shown in Fig. 8. Again, because of their long lifetimes, the agreement between the semiclassical and quantum resonance energies is of the order of $0.04 \mathrm{eV}$. That the two series of resonances belong to the same family is confirmed by the composition of the resonances (see Table II). This composition varies little within one series and is quite different from the other.

The different p.o.s associated with the resonances as well as the general features of the classical motion allows us to compare them to two other types of resonances found in other systems. In the $\mathrm{H}+\mathrm{H}_{2}$ collinear reactive scattering, two short-lived resonances appear, which are attributed to the short-lived p.o.s sitting on the dividing surface. ${ }^{19,53}$ As expected, these resonances are broad, and well reproduced by a simple semiclassical argument as ours. A somewhat different type of resonance has been observed in heavy-light-heavy systems, like $\mathrm{IH}+\mathrm{I}$ or the $\mathrm{H} \mu^{-} \mathrm{H}$ system. Because of kine- 


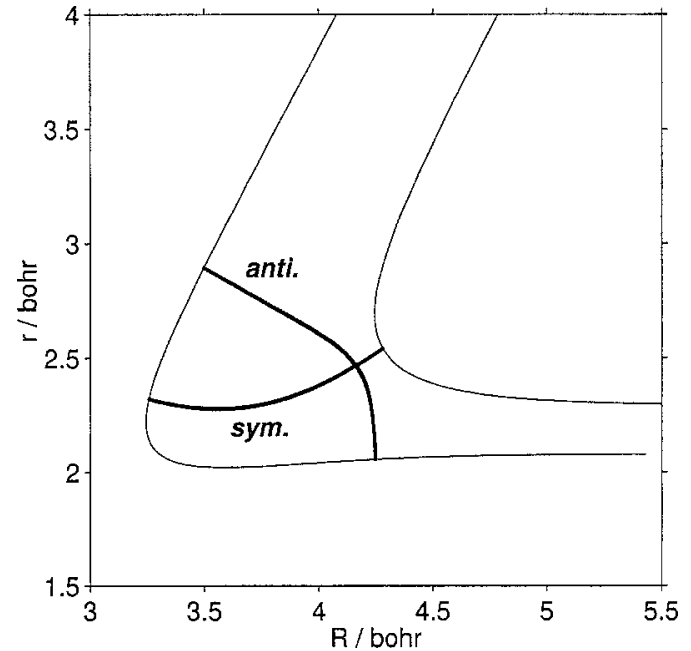

FIG. 6. Views of two stable periodic orbits of the LEPS PES, at an energy of $0.16 \mathrm{eV}$, along with the relevant equipotentials $V=0.16 \mathrm{eV} . r$ and $R$ are the mass-scaled Jacobi coordinates. Both periodic orbits are simple pods (periodic orbit dividing surface) in the sense of Ref. 41. They behave like symmetric and antisymmetric stretches.

matic effects, the light atom bounces back and forth in between the two heavy ones. In different contexts, this type of motion has been compared to the motion of the clapper inside a heavy bell. However, when momentum is transferred with the correct phase, the system finds its way out. ${ }^{53}$ This type of kinematics also gives rise to semiclassical resonances.

Here, like in the $\mathrm{HeH}_{2}^{+}$system, the resonances occur because of the vibrations of the CNO complex, whose lifetime is not short. Recall that, besides being exothermic, this reaction has no threshold, unlike $\mathrm{H}+\mathrm{H}_{2}$ one. The reactants reach directly the strong interaction region and the associated triatomic vibrational modes. Because of the moderate kinematic effect of the masses, the antisymmetric stretch is stable (both for the PQLEPS, the LEPS and other models). This

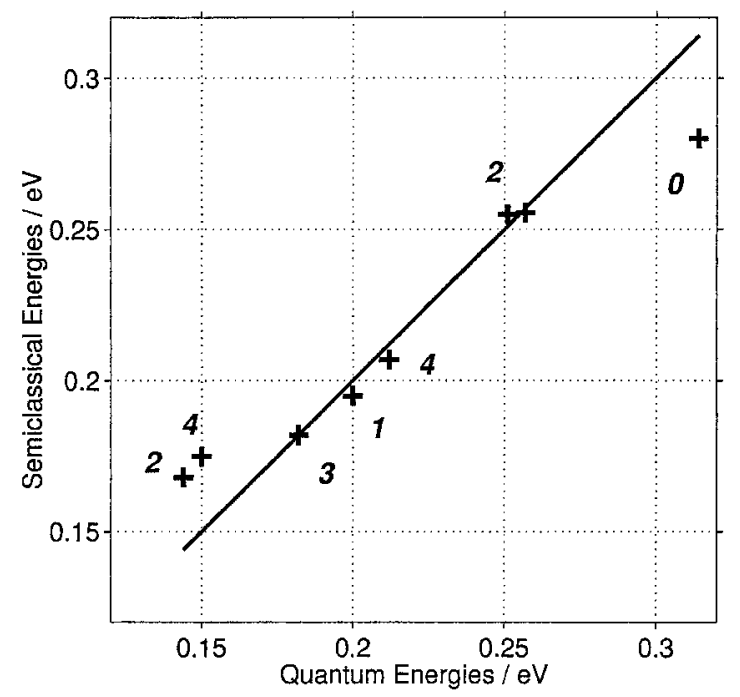

FIG. 7. Comparison between quantum and semiclassical resonance energies, for the PQLEPS PES. The numbers associated with the points refer to the periodic orbits of Fig. 5. The line having a slope of $45^{\circ}$ depicts the equation $E_{\text {quantum }}=E_{\text {semiclassical }}$.

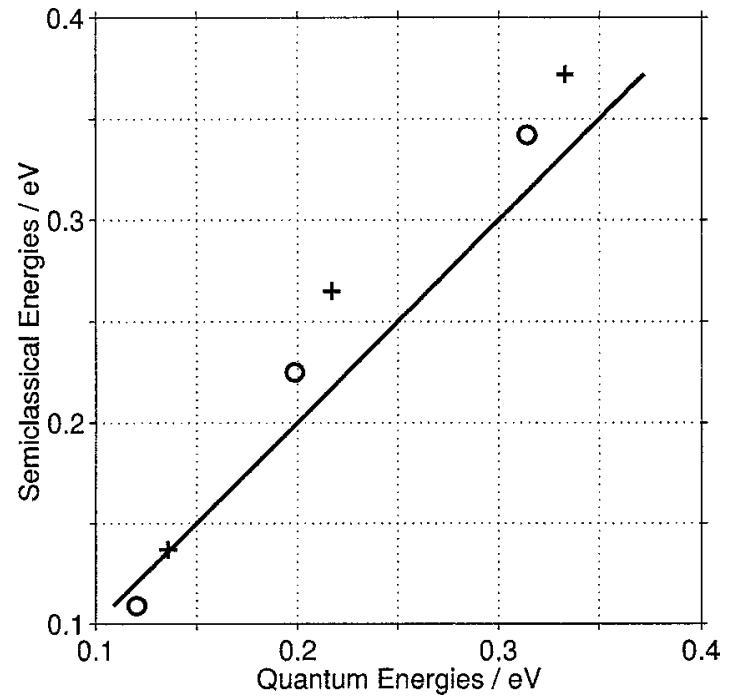

FIG. 8. Comparison between quantum and semiclassical resonance energies, for the LEPS PES. The circles denote the symmetric stretch periodic orbits, and the crosses, the antisymmetric stretch periodic orbits (see Fig. 6). The line having a slope of $45^{\circ}$ depicts the equation $E_{\text {quantum }}=E_{\text {semiclassical }}$.

vibration leads readily, at the low energies we are dealing with, to long-lived resonances. The PQLEPS PES has only one stable p.o., which is antisymmetric [see p.o. $\mathbf{0}$ in Fig. 5(a)]. This PES does not support any other family of simple long-lived vibrational modes, but supports instead a whole series of resonances, more or less short-lived $\left(q_{1}<0.7 \mathrm{ps}\right)$ [see Fig. 3(a)]. Classically speaking, the LEPS PES is indeed very similar to the PQLEPS one, as far as the p.o.s are concerned. However, the latter, with its deep well, supports fewer stable p.o.s, since a lot more kinetic energy is available in the region of this well. This argument seems to differ from a quantum one. As for a one-dimensional square well, one would expect quantum mechanically to observe longlived shape resonances in the PQLEPS PES due to the presence of the deep well. However, because of internal excitation mechanisms associated with couplings between motions along the transverse cut through this PES and asymmetric motions limited by the repulsive walls that are responsible for the skew angle of collinear PESs, one also expects the presence of Feshbach-type resonances (in both PESs). The "shape" processes in the PQLEPS PES seem to interfere with the Feshbach ones, leading to shorter-lived resonances than for the LEPS PES, for which the absence of a well essentially precludes shape-type mechanisms. This tentative interpretation requires further investigation. Nevertheless, the dominant factor for the PQLEPS and LEPS resonances, as compared with the $\mathrm{H}+\mathrm{H}_{2}$ ones, is the absence of a barrier (hence no repulsive pods) which induces several series of vibrational resonances of dynamical origin.

\section{SUMMARY AND CONCLUSIONS}

We have obtained scattering matrices for the collinear reaction $\mathrm{C}+\mathrm{NO} \rightarrow \mathrm{CN}+\mathrm{O}$, by performing accurate quantum mechanical reactive scattering calculations using two potential energy surfaces (PESs): a representative PQLEPS PES, which has a $4.26 \mathrm{eV}$ deep well in the strong interaction re- 
gion and an empirical LEPS PES, which has no well at all in that region. These scattering matrices were obtained at 2201 energies in the total energy range $0.12 \mathrm{eV}$ to $0.34 \mathrm{eV}$ with respect to the bottom of the isolated $\mathrm{NO}$ well [corresponding to a relative translational energy of $\mathrm{C}$ with respect to $\mathrm{NO}$ $(v=0)$ of $0.003 \mathrm{eV}$ to $0.223 \mathrm{eV}$ ] and used to obtain stateto-state reaction probabilities and collision lifetime matrices as a function of energy. The reaction probabilities versus energy curves for both PESs have pronounced structure that suggests the presence of resonances. These resonances were characterized by analyzing the eigenvalues and eigenvectors of the collision lifetime matrices. The observed resonances on the LEPS PES were longer-lived than those on the PQLEPS PES. The latter had more mixed compositions (4 to 7 major contributing asymptotic states) as compared to those on the LEPS PES (2 to 3 major contributing states) suggesting a role of the potential well in mixing the states. The results suggest that the presence of a potential well is not necessary for producing Feshbach-type internal excitation resonances, although it does affect their energies, lifetimes, and compositions. It also seems that when both shape and Feshbach processes are possible (as for the PQLEPS PES), they may interfere with each other.

We have also analyzed these resonances by a simple semiclassical WKB quantization, in terms of stable and unstable periodic orbits. These orbits were obtained for both PESs and the energies of the most stable ones were compared with the quantum resonance energies. Considering that a simple semiclassical quantization was used, a resonable agreement was obtained between these energies. Moreover, the origin of the resonances has been traced to some simple periodic orbits, stable and unstable. The large number of resonances is due to the existence of many long-lived periodic orbits in the strong interaction region, caused by the absence of a barrier in the PESs. This behavior contrasts with the one observed in many reactions having a barrier in that region, which results in an energy threshold for the reaction probabilities. The presence of a well is less important than the absence of a barrier in determining the characteristics of the resonances.

Although the results and analysis presented in this paper are based on collinear calculations, the low collision energies involved (of $0.003 \mathrm{eV}$ to $0.223 \mathrm{eV}$ ), together with the fact that the 3D PES of this system is collinearly-dominated suggests that related resonances and resonance mechanisms may be valid for the associated 3D system. In particular, it is quite possible that $3 \mathrm{D}$ resonances in this relatively heavy atom system may show up whether or not a deep well is present, and that their characteristics may be strongly influenced by the absence of a barrier. These possibilities suggest that a high quality 3D quantum calculation should be performed using a realistic PES as well as a modified one in which the deep well has been excised and perhaps a third one in which a barrier has been introduced. In any event, it would be very interesting to verify whether or not resonant behavior, which is a uniquely quantum property, is displayed in this and perhaps other heavy atom systems.

\section{ACKNOWLEDGMENTS}

One of us (L.W.) thanks Caltech for its welcome and support during the 1999-2000 academic year. This work has been supported in part by NSF Grant No. CHE 9810050 and by a joint NSF/CNRS Grant No. INT-9910196. The Laboratoire de Spectrométrie Physique is a joint CNRS/Université Joseph-Fourier laboratory, under the name UMR 5580.

${ }^{1}$ D. G. Truhlar and A. Kuppermann, J. Chem. Phys. 52, 3841 (1970).

${ }^{2}$ R. D. Levine and S. F. Wu, Chem. Phys. Lett. 11, 557 (1971).

${ }^{3}$ A. Kuppermann, in Potential Energy Surfaces and Dynamics Calculations, edited by D. G. Truhlar (Plenum, New York, 1981), pp. 375-420.

${ }^{4}$ D. N. Neumark, A. M. Wodke, G. N. Robinson, C. C. Hayden, and Y. T. Lee, Phys. Rev. Lett. 53, 226 (1984); J. Chem. Phys. 82, 3045 (1985).

${ }^{5}$ D. N. Neumark, A. M. Wodke, G. N. Robinson, C. C. Hayden, K. Shobotake, R. K. Sparks, T. P. Schafer, and Y. T. Lee, J. Chem. Phys. 82, 3067 (1985)

${ }^{6}$ A. Kuppermann and J. P. Dwyer, in Electronic and Atomic Collisions, Abstracts, edited by K. Takayanagi and N. Oda (Society for Atomic Collision Research (Japan), Tokyo, 1979), pp. 888-889.

${ }^{7}$ J. Römelt, Chem. Phys. 79, 197 (1983).

${ }^{8}$ E. Pollak, J. Chem. Phys. 76, 5843 (1982).

${ }^{9}$ A. Kuppermann and J. A. Kaye, J. Phys. Chem. 85, 1969 (1981).

${ }^{10}$ F. T. Smith, Phys. Rev. 118, 349 (1960).

${ }^{11}$ G. C. Schatz and A. Kuppermann, J. Chem. Phys. 59, 964 (1973).

${ }^{12}$ N. Abusalbi, D. J. Kouri, V. Lopez, V. K. Babamov, and R. A. Marcus, Chem. Phys. Lett. 103, 458 (1984).

${ }^{13}$ P. G. Hipes and A. Kuppermann, Chem. Phys. Lett. 133, 1 (1987).

${ }^{14}$ R. N. Porter and M. Karplus, J. Chem. Phys. 40, 1105 (1964).

${ }^{15}$ J. A. Kaye and A. Kuppermann, Chem. Phys. 127, 97 (1988).

${ }^{16}$ A. Kuppermann and Y.-S. M. Wu, Chem. Phys. Lett. 241, 229 (1995); 243, 229(E) (1995).

${ }^{17}$ N. Abusalbi, D. J. Kouri, M. Baer, and E. Pollak, J. Chem. Phys. 82, 4500 (1985).

${ }^{18}$ A. Rahaman and N. Sathyamurthy, J. Phys. Chem. 98, 12481 (1994); C. Kalyanaraman, D. C. Clary, and N. Sathyamurthy, J. Chem. Phys. 111, 10910 (1999); P. Bhatia, B. Maiti, N. Sathyamurthy, S. Stamatiadis, and S. C. Farantos, Phys. Chem. Chem. Phys. 1, 1105 (1999).

${ }^{19}$ M. S. Child, Semiclassical Mechanics with Molecular Applications (Oxford University Press, Oxford, 1991).

${ }^{20}$ R. Sadeghi and R. T. Skodje, J. Chem. Phys. 99, 5126 (1993).

${ }^{21}$ R. Sadeghi and R. T. Skodje, J. Chem. Phys. 102, 193 (1995)

${ }^{22}$ S. Garashchuk, F. Grossmann, and D. Tannor, J. Chem. Soc., Faraday Trans. 93, 781 (1997).

${ }^{23}$ G. Campolieti and P. Brumer, J. Chem. Phys. 109, 2999 (1998).

${ }^{24}$ P. Gaspard and I. Burghardt, Adv. Chem. Phys. 101, 491 (1997).

${ }^{25}$ N. De Leon and E. Heller, J. Chem. Phys. 81, 5957 (1984).

${ }^{26}$ C. Clay Marston, J. Chem. Phys. 103, 8456 (1995).

${ }^{27}$ R. Prosmiti, S. C. Farantos, R. Guantes, F. Borondo, and R. M. Benito, J. Chem. Phys. 104, 2921 (1996).

${ }^{28}$ H. Ishikawa, R. W. Field, S. C. Farantos, M. Joyeux, J. Koput, C. Beck, and R. Schinke, Annu. Rev. Phys. Chem. 50, 443 (1999).

${ }^{29}$ A. Wirzba and M. Henseler, J. Phys. A 31, 2155 (1998).

${ }^{30}$ P. Halvick, J. C. Rayez, and E. M. Evleth, J. Chem. Phys. 81, 728 (1984).

${ }^{31}$ P. Halvick, J. C. Rayez, M. T. Rayez, and B. Duguay, Chem. Phys. 114, 375 (1987).

${ }^{32}$ J. C. Rayez, P. Halvick, M. T. Rayez, and B. Duguay, Chem. Phys. 101, 401 (1986).

${ }^{33}$ P. Halvick and J. C. Rayez, Chem. Phys. 131, 375 (1989).

${ }^{34}$ L. Bonnet, J. C. Rayez, and P. Halvick, Chem. Phys. Lett. 216, 11 (1993).

${ }^{35}$ M. Monnerville, P. Halvick, and J. C. Rayez, J. Chem. Soc., Faraday Trans. 89, 1579 (1993).

${ }^{36}$ E. Wallin, P.-A. Elofson, and L. Holmlid, Chem. Phys. 163, 37 (1992); 185, 91 (1994).

${ }^{37}$ A. Beghin, T. Stoecklin, and J. C. Rayez, Chem. Phys. 195, 259 (1995).

${ }^{38}$ B. J. Persson, B. O. Roos, and M. Simonson, Chem. Phys. Lett. 234, 382 (1995).

${ }^{39}$ B. J. Persson, B. O. Roos, and S. Carter, Mol. Phys. 84, 619 (1995).

${ }^{40}$ M. Simonson, N. Markovic, S. Nordholm, and B. J. Persson, Chem. Phys. 200, 141 (1995). 
${ }^{41}$ H. Wadi and L. Wiessenfeld, Chem. Phys. Lett. 300, 37 (1999).

${ }^{42}$ S. Andersson, N. Maraković, and G. Nyman, Phys. Chem. Chem. Phys. 2, 613 (2000).

${ }^{43}$ M. Monnerville, G. Péoux, S. Briquez, and P. Halvick, Chem. Phys. Lett. 322, 157 (2000).

${ }^{44}$ A. Kuppermann, J. A. Kaye, and J. P. Dwyer, Chem. Phys. Lett. 74, 257 (1980).

${ }^{45}$ M. Brack and R. K. Bhaduri, Semiclassical Physics (Addison-Wesley, Reading, 1997).

${ }^{46}$ M. V. Berry and M. Tabor, Proc. R. Soc. London, Ser. A 349, 101 (1976).
${ }^{47}$ M. C. Gutzwiller, Chaos in Classical and Quantum Mechanics (SpringerVerlag, New York, 1990).

${ }^{48}$ A. Voros, Prog. Theor. Phys. Suppl. 116, 17 (1994).

${ }^{49}$ G. Tanner, K. T. Hansen, and G. Main, Nonlinearity 9, 1641 (1996).

${ }^{50}$ R. O. Vallejos, A. M. Ozorio de Almeida, and C. H. Lewenkopf, J. Phys. A 31, 4885 (1998).

${ }^{51}$ S. C. Creagh, J. M. Robbins, and R. G. Littlejohn, Phys. Rev. A 42, 1907 (1990).

${ }^{52}$ Z. Kovács and L. Wiesenfeld, Phys. Rev. E 51, 5476 (1995).

${ }^{53}$ E. Pollak, in Theory of Chemical Reaction Dynamics, edited by M. Baer (CRC, Boca Raton, 1985), Vol. III, pp. 123-246. 